\title{
Macro Broth Dilution Method
}

National Cancer Institute

\section{Source}

National Cancer Institute. Macro Broth Dilution Method. NCI Thesaurus. Code C85697.

A method to determine the minimum inhibitory concentration (MIC) of antibiotics in which growth media is pipetted into a test tube. Each tube contains a known concentration of drug and is inoculated with a known quantity of bacteria. The tubes are incubated for a specified period of time and each is assessed for bacterial cell growth. The drug concentration in the first tube showing no growth is known as the MIC for that antibiotic. 